\title{
Identification of Potential Serum Exosomal microRNAs Involved in Acinar-Ductal Metaplasia That is A Precursor of Pancreatic Cancer Associated with Chronic Pancreatitis
}

\author{
Li-Ping Sheng \\ Wuhan Union Hospital \\ Chao-Qun Han \\ Wuhan Union Hospital \\ Chi Nie \\ Wuhan Union Hospital \\ Tao Xu \\ Wuhan Union Hospital \\ Kun Zhang \\ Wuhan Union Hospital \\ Xuan-Ji Li \\ Wuhan Union Hospital \\ Xin-Ru Xie \\ Wuhan Union Hospital \\ Rong Lin \\ Wuhan Union Hospital \\ Zhen Ding ( $\nabla 2645176131 @ q q . c o m$ ) \\ Wuhan Union Hospital https://orcid.org/0000-0002-1128-0875
}

Research article

Keywords: miRNA, exosome, chronic pancreatitis, acinar-ductal metaplasia, bioinformatics analysis

Posted Date: July 13th, 2020

DOI: https://doi.org/10.21203/rs.3.rs-40982/v1

License: () (1) This work is licensed under a Creative Commons Attribution 4.0 International License.

Read Full License 


\section{Abstract}

Backgrounds Due to difficulty in early diagnosis of chronic pancreatitis (CP), it is urgent to find novel biomarkers to detect CP. Exosomal microRNAs (Exo-miRNAs) located in the serum may be potential diagnostic and therapeutic targets for CP. In our study, we performed a bioinformatics analysis to identify differentially expressed Exo-miRNAs (DE-Exo-miRNAs) in the serum of CP patients.

Methods The dataset GSE128508 was downloaded from the Gene Expression Omnibus (GEO) database. The analysis was carried out using BRB-ArrayTools and Significance Analysis of Microarrays (SAM). The target genes of DE-S-Exo-miRNAs were predicted by miRWalk databases. Further gene ontology (GO) term and Kyoto Encyclopedia of Genomes (KEGG) pathway analyses were performed with plug-in ClueGO in Cytoscape software 3.7.0. Subsequently, the interaction regulatory network between encoded proteins of target genes was performed with the Search Tool for the Retrieval of Interacting Genes (STRING) database and analyzed using plug-in MCODE and cytoHubba in Cytoscape software 3.7.0.

Results We identified 227 DE-Exo-miRNAs in the serum. Further analysis using the miRWalk database identified 5164 target genes of these miRNAs. The protein-protein interaction (PPI) regulatory network of 1912 potential target genes for hub 10 up-regulated miRNAs with high degrees and one down-regulated miRNAs were constructed using the STRING database and Cytoscape software. The functional analysis using Cytoscape software tool highlighted that target genes involved in pancreatic cancer. Acinar-ductal metaplasia (ADM) in the inflammatory environment of $\mathrm{CP}$ is a precursor of pancreatic cancer. Subsequently, we constructed a network of target genes associated with ADM and their miRNAs.

Conclusions Exo-miRNAs in the serum as well as their target genes may be promising targets for the early diagnosis and treatment of CP. In addition, we identified potential Exo-miRNAs involved in ADM that is a precursor of pancreatic cancer associated with $\mathrm{CP}$.

\section{Introduction}

Chronic pancreatitis (CP) is a form of continuing fibro-inflammatory disease associated with pathologically morphological changes of the pancreas, typically causing endocrine and exocrine dysfunction, especially in persons carrying genetic risk factors [1]. The diagnosis of CP can be difficulty in early phases of disease. Here, pancreatic functions are preserved and tests of laboratory and imaging may only display minimally abnormal results [2]. Thus, a comprehensive understanding about the molecular mechanism underlying $\mathrm{CP}$ and the identification of novel targets to diagnose $\mathrm{CP}$ in early phase are necessary.

MicroRNAs (miRNAs) are associated with acute or chronic pancreatitis [3-7]. miRNAs are single-stranded RNAs 21-23-nucleotides in length which have important functions in almost every aspect of biology [8]. miRNAs play crucial gene regulatory roles by directing post-transcriptional repression of proteincoding genes [9]. The roles of miRNAs in many human diseases have made them promising targets for 
diagnostic and therapeutic strategies [10-12]. miRNAs have been discovered in body fluids, such as serum and plasma $[13,14]$. Additionally, researchers have also extracted miRNAs from exosomes [15].

Exosomes are formed by the fusion of multivesicular bodies with the plasma membrane and are then released from the cell into the microenvironment [16]. Exosomes regulate many pathophysiological processes, such as inflammation, infection, immune responses, and tumor growth [17]. Exosomes contain proteins, lipid and miRNAs $[18,19]$. As miRNAs in exosomes are prevented from RNase degradation, they are easier to detect compared to freely circulating miRNAs [20]. In addition, the miRNA expression profile in exosomes is different compared to the corresponding intracellular miRNAs [21]. Acute pancreatitis plasma exosomes contained more pro-inflammatory miRNA than exosomes from plasma control [22]. Therefore, exosomal microRNAs (Exo-miRNAs) in the serum could be potential biomarkers for diagnosing $\mathrm{CP}$.

In this study, we analyzed the dataset GSE128508 from the public Gene Expression Omnibus (GEO) database. The samples displayed in the dataset are from the serum exosomes from CP patients, autoimmune pancreatitis (AIP) patients, and normal participants. Previous study focused on AIP and identified 10 differentially expressed Exo-miRNAs (DE-Exo-miRNAs) [23]. We obtained more interesting DE-Exo-miRNAs in the serum by only comparing CP patients and normal participants using Significance Analysis of Microarrays (SAM). Subsequently, functional analysis of target genes for DE-Exo-miRNAs from the miRWalk databases was performed using Cytoscape software tool. Then the target genes of 10 up-regulated miRNAs with high degrees and one down-regulated miRNAs selected from miRNAs-mRNA networks were used to construct a protein-protein interaction (PPI) regulatory network. As functionally grouped network of KEGG pathways show that pancreatic cancer is significant and acinar-ductal metaplasia (ADM) in the inflammatory environment of $C P$ is a precursor of pancreatic cancer [24], the regulatory network of target genes associated with ADM and their DE-Exo-miRNAs in the serum were also constructed.

\section{Materials And Methods \\ Data acquisition}

In this study, we retrieve datasets from public GEO database. The search keyword was 'pancreatitis' and the selection was restricted to series. Finally, the miRNA dataset GSE128508 using the GPL21263 platform, which included results from $10 \mathrm{CP}$ and 10 healthy adults' samples, were downloaded for further analyses.

\section{Data processing and identifying serous DE-Exo-miRNAs}

The data from GSE128508 were analyzed by BRB-ArrayTools and SAM to identify serous DE-Exo-miRNAs between $\mathrm{CP}$ and normal samples. We set the minimum fold change $=1$ and Delta $=0.01$ as threshold.

\section{Identification of serous DE-Exo-miRNA target genes}


The miRWalk database is a large collection of predicted and experimentally verified binding sites information for miRNAs-targets [25]. The information for DE-Exo-miRNA target genes was obtained from the miRWalk databases. We selected genes from the intersection between the Targetscan and miRDB databases, as well as experimentally verified miRTarBase database to increase the accuracy of the predictions.

\section{Function analysis of target genes}

Potential functions of target genes in biological process were analyzed using GO term. KEGG is a database for the systematic analysis of gene functions and utilities in biological system from molecularlevel information. We obtained information from both the GO term and KEGG pathway using the plug-in ClueGO (2.5.6) in Cytoscape software 3.7.0 [26].

\section{Construction of miRNA-mRNA networks and miRNAs screening}

Many gene targets of DE-Exo-miRNA in the serum may be associated with CP. The miRNA-mRNA networks were visualized with Cytoscape software 3.7.0. We identified hub miRNAs in networks according to their intra-network connectivity and correlation with their target genes. The10 up-regulated miRNAs with high degrees and one down-regulated miRNAs were identified from the networks.

\section{Construction of PPI networks and significant submodule screening}

The functional interactions between encoded proteins of target genes for 11 dysregulated miRNAs were explored using STRING database [27]. We downloaded the interaction information from the STRING database and use Cytoscape software 3.7.0 to construct the PPI regulatory network. We applied the MCODE tool of Cytoscape software 3.7.0 to identify the significant submodule from the PPI network [28]. Furthermore, the hub genes were identified using cytoHubba plugin of Cytoscape software 3.7.0 from the significant submodule [29]. We set degree cutoff $=2$, node score cutoff $=0.2, \mathrm{~K}$-core $=2$, and maximum depth $=100$ as the thresholds.

\section{Construction of the regulatory network associated with ADM}

KEGG pathways analysis involved in pancreatic cancer and pathways in cancer. Pancreas acinar cells show high plasticity and can undergo ADM after injury. Cells undergoing ADM are precursors for pancreatic intraepithelial neoplasia lesions that can further progress to pancreatic ductal adenocarcinoma (PDAC) [30]. Among target genes of DE-Exo-miRNAs in the serum, signal transducer and activator of transcription 3 (STAT3), 3-phosphoinositide-dependent protein kinase 1 (PDK1), Kirsten rat sarcoma 2 viral oncogene homolog (KRAS), Ras-related C3 botulinum toxin substrate 1 (RAC1), and Ras homolog family member $\mathrm{A}(\mathrm{RHOA})$ are key factors involving in ADM [31-34]. Therefore, we construct the regulatory network of these target genes and their DE-Exo-miRNAs. 


\section{Results}

\section{DE-Exo-miRNAs in CP serum}

A total of 227 DE-Exo-miRNAs in the serum were identified from the dataset GSE128508. Among these DE-Exo-miRNAs, 226 were significantly up-regulated and one were down-regulated. One down-regulated DE-Exo-miRNAs and the top 50 up-regulated DE-Exo-miRNAs in the serum can be found summarized in Table 1. In supplementary materials, all 227 DE-Exo-miRNAs in the serum are listed. 
Table 1

List of partial DE-Exo-miRNAs in the serum*

\section{Score Numerator Denominator Fold Change}

Down-regulated microRNAs
hsa-miR-5100
$-4.20$
$-1.32$
0.31
0.82

Up-regulated microRNAs (Top 50)

\begin{tabular}{|lllll|}
\hline hsa-miR-5006-5p & 13.60 & 1.65 & 0.12 & 1.50 \\
\hline hsa-miR-6748-5p & 13.56 & 2.11 & 0.16 & 1.63 \\
\hline hsa-miR-4485-5p & 12.34 & 1.74 & 0.14 & 1.52 \\
\hline hsa-miR-5192 & 11.75 & 2.34 & 0.20 & 1.70 \\
\hline hsa-miR-4514 & 11.73 & 2.22 & 0.19 & 1.67 \\
\hline hsa-miR-6760-5p & 11.36 & 2.83 & 0.25 & 1.77 \\
\hline hsa-miR-6165 & 10.97 & 2.44 & 0.22 & 1.63 \\
\hline hsa-miR-4298 & 10.95 & 2.44 & 0.22 & 1.63 \\
\hline hsa-miR-6846-5p & 10.92 & 2.19 & 0.20 & 1.62 \\
\hline hsa-miR-6887-5p & 10.91 & 3.26 & 0.30 & 1.78 \\
\hline hsa-miR-6830-5p & 10.83 & 1.71 & 0.16 & 1.51 \\
\hline hsa-miR-6828-5p & 10.82 & 1.97 & 0.18 & 1.59 \\
\hline hsa-miR-370-3p & 10.68 & 2.50 & 0.23 & 1.69 \\
\hline hsa-miR-6766-5p & 10.65 & 2.31 & 0.22 & 1.61 \\
\hline hsa-miR-3151-5p & 10.64 & 1.95 & 0.18 & 1.57 \\
\hline hsa-miR-6795-5p & 10.60 & 2.20 & 0.21 & 1.54 \\
\hline hsa-miR-3622b-5p & 10.50 & 1.66 & 0.16 & 1.50 \\
\hline hsa-miR-4419b & 10.49 & 2.51 & 0.24 & 1.61 \\
\hline hsa-miR-513a-5p & 10.41 & 2.01 & 0.19 & 1.61 \\
\hline hsa-miR-3154 & 10.41 & 2.11 & 0.20 & 1.55 \\
\hline hsa-miR-6826-5p & 10.22 & 2.01 & 0.20 & \\
\hline
\end{tabular}

*: All 227 DE-Exo-miRNAs in the serum are listed in supplementary materials. DE-Exo-miRNAs: differentially expressed exosomal miRNAs, Score: The T-statistic value, Numerator: The numerator of the T-statistic, Denominator: The denominator of the T-statistic. 


\begin{tabular}{|c|c|c|c|c|}
\hline & Score & Numerator & Denominator & Fold Change \\
\hline hsa-miR-4728-5p & 10.08 & 2.81 & 0.28 & 1.66 \\
\hline hsa-miR-4727-3p & 9.93 & 1.76 & 0.18 & 1.51 \\
\hline hsa-miR-3619-3p & 9.87 & 2.74 & 0.28 & 1.49 \\
\hline hsa-miR-6849-5p & 9.86 & 2.34 & 0.24 & 1.56 \\
\hline hsa-miR-6717-5p & 9.82 & 2.74 & 0.28 & 1.60 \\
\hline hsa-miR-5010-5p & 9.81 & 1.68 & 0.17 & 1.50 \\
\hline hsa-miR-491-5p & 9.77 & 1.65 & 0.17 & 1.50 \\
\hline hsa-miR-6746-5p & 9.69 & 2.29 & 0.24 & 1.51 \\
\hline hsa-miR-6890-5p & 9.66 & 1.87 & 0.19 & 1.54 \\
\hline hsa-miR-6824-5p & 9.55 & 2.13 & 0.22 & 1.53 \\
\hline hsa-miR-6757-5p & 9.54 & 2.51 & 0.26 & 1.54 \\
\hline hsa-miR-2110 & 9.53 & 1.80 & 0.19 & 1.50 \\
\hline hsa-miR-4257 & 9.51 & 2.13 & 0.22 & 1.48 \\
\hline hsa-miR-6731-5p & 9.44 & 1.86 & 0.20 & 1.52 \\
\hline hsa-miR-3714 & 9.40 & 1.93 & 0.21 & 1.54 \\
\hline hsa-miR-4462 & 9.29 & 1.95 & 0.21 & 1.50 \\
\hline hsa-miR-920 & 9.28 & 2.58 & 0.28 & 1.53 \\
\hline hsa-miR-7846-3p & 9.10 & 2.19 & 0.24 & 1.55 \\
\hline hsa-miR-7847-3p & 9.08 & 2.43 & 0.27 & 1.55 \\
\hline hsa-miR-6812-5p & 9.00 & 2.04 & 0.23 & 1.53 \\
\hline hsa-miR-3934-5p & 8.84 & 1.90 & 0.21 & 1.49 \\
\hline hsa-miR-583 & 8.83 & 1.45 & 0.16 & 1.44 \\
\hline hsa-miR-5189-5p & 8.83 & 1.83 & 0.21 & 1.51 \\
\hline hsa-miR-6777-5p & 8.71 & 2.28 & 0.26 & 1.51 \\
\hline hsa-miR-6511b-5p & 8.68 & 1.79 & 0.21 & 1.47 \\
\hline hsa-miR-8059 & 8.66 & 2.29 & 0.26 & 1.46 \\
\hline
\end{tabular}




\begin{tabular}{|lllll|}
\hline & Score & Numerator & Denominator & Fold Change \\
\hline hsa-miR-551b-5p & 8.61 & 1.83 & 0.21 & 1.51 \\
\hline hsa-miR-6769b-5p & 8.61 & 1.79 & 0.21 & 1.43 \\
\hline hsa-miR-6756-5p & 8.59 & 2.03 & 0.24 & 1.36 \\
\hline $\begin{array}{l}\text { *: All 227 DE-Exo-miRNAs in the serum are listed in supplementary materials. DE-Exo-miRNAs: } \\
\text { differentially expressed exosomal miRNAs, Score: The T-statistic value, Numerator: The numerator of } \\
\text { the T-statistic, Denominator: The denominator of the T-statistic. }\end{array}$ & \\
\hline
\end{tabular}

\section{Target genes for serous DE-Exo-miRNAs}

We utilized the miRWalk database to screen for target genes for the 227 DE-Exo-miRNAs in the serum to explore the potential functions of these DE-Exo-miRNAs. To increase the accuracy of the search, the intersection between the TargetScan and miRDB databases, as well as experimentally verified genes in the miRTarBase database, were selected as target genes. Finally, we obtained 5164 target genes for the 227 serous DE-Exo-miRNAs.

\section{Function analysis of target genes for serous DE-Exo- miRNAs}

The function analysis indicated that the enriched GO terms of 5164 target genes primarily included those involved in the cell cycle process, cellular response to signal transduction by p53 class mediator, lymphocyte mediated immunity, regulation of cell death, regulation of cell differentiation, regulation of gene expression, regulation of cell communication, regulation of cellular metabolic process, and regulation of B cell activation, amongst others. The typical $20 \mathrm{GO}$ terms are summarized in Fig. 1.

The KEGG pathways analysis revealed that target genes were involved in numerous different pathways, including pancreatic cancer, pathways in cancer, p53 signaling pathway, mitogen-activated protein kinase (MAPK) signaling pathway, cellular senescence, and signaling pathways regulating pluripotency of stem cells, amongst others. These pathways are shown in Fig. 2.

\section{The serous DE-Exo-miRNAs with high degrees}

We constructed the miRNAs-mRNA networks between the 227 DE-Exo-miRNAs and 5164 target genes using Cytoscape software 3.7.0. The hub 10 up-regulated miRNAs with high degrees identified from the miRNAs-mRNA networks are summarized in Table 2. 
Table 2

The top 10 miRNAs with high degrees from miRNAs-mRNA networks.

\begin{tabular}{|lllll|}
\hline Rank & miRNA & Degree & Number of target genes & Feature \\
\hline 1 & hsa-miR-24-3p & 516 & 516 & up \\
\hline 2 & hsa-miR-149-3p & 449 & 449 & up \\
\hline 3 & hsa-miR-6785-5p & 448 & 448 & up \\
\hline 4 & hsa-miR-4728-5p & 446 & 446 & up \\
\hline 5 & hsa-miR-6808-5p & 402 & 402 & up \\
\hline 6 & hsa-miR-6779-5p & 388 & 388 & up \\
\hline 7 & hsa-miR-6799-5p & 384 & 384 & up \\
\hline 8 & hsa-miR-6086 & 343 & 343 & up \\
\hline 9 & hsa-miR-4722-5p & 286 & 286 & up \\
\hline 10 & hsa-miR-4433a-3p & 245 & 245 & \\
\hline $\begin{array}{l}\text { Degree stands for miRNA intra-network connectivity and correlation with their target genes. The } \\
\text { miRNA with most high-degree are the most hub node in the network. }\end{array}$
\end{tabular}

\section{The Significant submodule from the PPI regulatory network}

The 10 up-regulated miRNAs, one down-regulated miRNAs (hsa-miR-5100), and their 1912 target genes were explored further. Using the interaction information of the target genes obtained from the STRING database, the PPI regulatory network of 1912 target genes for 11 key serous DE-Exo-miRNAs were constructed. We selected a significant submodule with the highest scores of 31.226 from the PPI regulatory network (Fig. 3). Furthermore, hub genes were identified from the significant submodule, including Von Hippel-Lindau disease tumor suppressor (VHL), E3 ubiquitin-protein ligase NEDD4-like (NEDD4L), Ubiquitin-conjugating enzyme E2 N (UBE2N), ubiquitin-conjugating enzyme E2 B (UBE2B), Fbox/WD repeat-containing protein $1 \mathrm{~A}$ (BTRC), Ubiquitin conjugating enzyme E2 D3 (UBE2D3), Ubiquitinconjugating enzyme E2 K (UBE2K), E3 ubiquitin-protein ligase Arkadia (RNF111), Cyclin-F (CCNF), Ubiquitin-conjugating enzyme E2 S (UBE2S).

\section{Regulatory network of target genes associated with ADM and their miRNAs}

Among the target genes of DE-Exo-miRNAs in the serum, STAT3, PKD1, KRAS, RAC1, and RHOA are key factors participating in ADM. miRNAs play crucial gene regulatory roles by directing post-transcriptional repression of protein-coding genes. The expression of these target genes may be repressed by the DE-SExo-miRNAs in the serum (Fig. 4). 


\section{Discussion}

The molecular mechanisms of $\mathrm{CP}$ are complex and diagnosis can be difficulty in early phases of the disease [2]. Therefore, it is of great importance to understand the molecular mechanism of CP, as well as identify new targets to diagnose CP. Exo-miRNAs in the serum could be effective biomarkers for CP diagnosis, as they are stable and informative. Here, we downloaded the publicly accessible CP patient dataset GSE128508 and performed bioinformatic analysis. Previous study focused on AIP and only identified 10 differentially expressed Exo-miRNAs (DE-Exo-miRNAs) [23]. When only comparing CP and normal samples, we identified 227 interesting DE-Exo-miRNAs in the serum. Moreover, we identified 5164 target genes of the 227 dysregulated Exo-miRNAs in CP serum. GO term and KEGG pathway analysis of these 5164 target genes indicated that they involved in the regulation of numerous biological processes and signaling pathways such as pancreatic cancer, amongst many others. ADM in the inflammatory environment of pancreatitis is a precursor of pancreatic cancer. The expression of the target genes associated with ADM (STAT3, PKD1, KRAS, RAC1, and RHOA) may be repressed by DE-Exo-miRNAs in the serum, which may contribute to blocking ADM initiation.

GO term analysis indicated that these target genes were enriched in the cell cycle process, cellular response to signal transduction by p53 class mediator, lymphocyte mediated immunity, regulation of cell death, regulation of cell differentiation, regulation of gene expression, regulation of cell communication, regulation of cellular metabolic process, and regulation of B cell activation, amongst others. These biological processes are associated with CP. Cyclin D1, an important regulator of the G1 phase of the cell cycle, increases in the pancreas of patients with $\mathrm{CP}$, which may contribute to a hyperproliferative state in $\mathrm{CP}$ [35]. Inhibition of p53 expression decreased levels of inflammatory factors and suppressed progression of CP in the mouse model [36]. The chronic inflammatory process in CP markedly affects immune function in CP patients [37]. Pancreatitis also leads cell death, such as apoptosis and necrosis [38]. Pancreatic acinar cells undergoing ADM contribute to the regeneration of the pancreas in response to injury [39]. The target genes of miRNA in serum exosomes may promote ADM by regulation of cell differentiation and gene expression. Cell communication of pancreatic acinar cells is associated with basal release of amylase [40]. The disruption of the metabolism at a molecular level occurs in the pancreas during pancreatitis [41]. Finally, the pancreatic B cell can secrete insulin [42]. The target genes of miRNA in serum exosomes may remedy or exacerbate diabetes mellitus (DM) secondary to chronic pancreatitis by regulation of $B$ cell activation.

The information regarding KEGG pathway analysis indicated that the target genes involve in pancreatic cancer, pathways in cancer, p53 signaling pathway, mitogen-activated protein kinase (MAPK) signaling pathway, cellular senescence, and signaling pathways regulating pluripotency of stem cells, amongst others. Chronic pancreatitis increases the risk of pancreatic cancer [43]. p53 is one of the most important tumor suppressor genes that is frequently mutated in human cancers [44]. p53 mutations can be detected in some cases of CP [45]. The target genes associated with pancreatic cancer, pathways in cancer, and p53 signaling pathway may contribute to pancreatic cancer associated with CP. As the major effector cells, activation of pancreatic stellate cells (PSCs) contributes to fibrosis of PC [46]. PSCs can be 
activated by many cellular signals, such as MAPK pathway [47]. The number of senescent PSCs are associated with the severity of inflammation and the extension of fibrosis of CP [48]. Pancreatic acinar cells show plasticity and dedifferentiate to an embryonic progenitor phenotype in experimental chronic pancreatitis [49]. Signaling pathways regulating pluripotency of stem cells may involve in this process. Therefore, a comprehensive understanding of the KEGG pathway analysis could be helpful in exploring complicated mechanisms underlying $\mathrm{CP}$.

We selected the top 10 up-regulated miRNAs with high degrees and one up-regulated miRNAs to constructed a PPI regulatory network and selected a significant submodule with the highest scores from the PPI regulatory network. Furthermore, hub genes were identified from the significant submodule. Our current understanding for the functions of hue genes in CP are limited. As tumor suppressor gene, VHL inhibits the proliferation, migration and invasion of pancreatic cancer cells [50]. Moreover, UBE2S regulates pancreatic cancer cell metastasis and invasion by epithelial-mesenchymal transition [51]. VHL and UBE2S may involve in pancreatic cancer associated with CP. The roles of other hub genes in CP need to be researched further in future studies.

Functionally grouped network of KEGG pathways show that pancreatic cancer and pathways in cancer are significant. $A D M$ in the inflammatory environment of $C P$ is a precursor of pancreatic cancer. Among the target genes of DE-Exo-miRNAs in the serum, STAT3, PKD1, KRAS, RAC1, and RHOA are key factors participating in ADM. STAT3 and PDK1 are critical effectors of oncogenic KRAS in the pancreas, mediating ADM formation [31, 32]. Activation of Rac1 is required for transdifferentiation of acinar cells into ADM [33]. Moreover, activation of RhoA/ROCK signaling pathway promotes the formation of ADM [34]. We construct the regulatory network of these target genes and their DE-Exo-miRNAs in the serum. Nevertheless, the relationship between DE-Exo-miRNAs and target genes associated with ADM require future experimental verification and could provide interesting therapeutic leads of preventing pancreatic cancer associated with $\mathrm{CP}$.

As a class of non-coding RNAs, one major function of miRNAs is the post-transcriptional repression of their target genes and they may be potential therapeutic targets for CP by interfering with the relative genetic factors [52]. miRNA-21 and connective tissue growth factor (CCN2) in PSC-derived exosomes are newly identified participants in PSC fibrogenic signaling during CP [53]. The exosomes may easily transport miRNAs to their target cells by encapsulating and protecting them. Therefore, studying the functions of DE-Exo-miRNAs in the serum and target genes is a promising avenue to identifying novel therapeutics.

In our work, we use bioinformatics analysis to identify serous DE-Exo-miRNAs between CP and normal samples. We then obtained their target genes from the miRWalk database. Functional analysis of these target genes as well as interactions between them provided insights into the molecular mechanism of CP. We constructed a network of target genes associated with ADM and their DE-Exo-miRNAs in the serum, as the interactions between these could likely underlie ADM in CP that is a precursor of pancreatic cancer. 
Nevertheless, further experimental validation is required for these predictions, which was a limitation for our study.

In summary, Exo-miRNAs in the serum and their target genes may be potential tools for the early diagnosis and treatment of CP. In addition, we identified potential Exo-miRNAs involved in ADM that is a precursor of pancreatic cancer associated with $\mathrm{CP}$.

\section{Declarations}

\section{Conflicts of interest}

The authors disclose no conflicts of interest.

\section{Funding}

This study was supported in part by the National Natural Science Foundation of China (81770637).

\section{Authors contribution}

Ding Zhen, Li-Ping Sheng and Chao-Qun Han participate in the concept and design of this study as well as drafting the manuscript. Li-Ping Sheng and Chao-Qun Han performed the data acquisition, the data analysis and interpretation. All authors have read and approved the manuscript.

\section{References}

1. Whitcomb DC, Frulloni L, Garg P, Greer JB, Schneider A, Yadav D, Shimosegawa T. Chronic pancreatitis: An international draft consensus proposal for a new mechanistic definition. Pancreatology. 2016;16(2):218-24.

2. Gupte A, Goede D, Tuite R, Forsmark CE. Chronic pancreatitis. BMJ. 2018;361:k2126.

3. Yu P, Liu K, Gao X, Karmouty-Quintana H, Bailey JM, Cao Y, Ko TC. Transforming Growth Factor- $\beta$ and Bone Morphogenetic Protein 2 Regulation of MicroRNA-200 Family in Chronic Pancreatitis. Pancreas. 2018;47(2):252-6.

4. Wang Z, Li D, Jin J, Wang Q, Zhao S, Bai Y. Association between microRNA polymorphisms and chronic pancreatitis. Pancreatology. 2016;16(2):244-8.

5. Xin L, Gao J, Wang D, Lin J-H, Liao Z, Ji J-T, Du T-T, Jiang F, Hu L-H, Li Z-S. Novel blood-based microRNA biomarker panel for early diagnosis of chronic pancreatitis. Sci Rep. 2017;7:40019.

6. Wang D, Xin L, Lin J-H, Liao Z, Ji J-T, Du T-T, Jiang F, Li Z-S, Hu L-H. Identifying miRNA-mRNA regulation network of chronic pancreatitis based on the significant functional expression. Med (Baltim). 2017;96(21):e6668. 
7. Fan L, Hui X, Mao Y, Zhou J. Identification of Acute Pancreatitis-Related Genes and Pathways by Integrated Bioinformatics Analysis. Dig Dis Sci 2019.

8. Li Z, Rana TM. Therapeutic targeting of microRNAs: current status and future challenges. Nat Rev Drug Discov. 2014;13(8):622-38.

9. Bartel DP. MicroRNAs: target recognition and regulatory functions. Cell. 2009;136(2):215-33.

10. Rupaimoole R, Slack FJ. MicroRNA therapeutics: towards a new era for the management of cancer and other diseases. Nat Rev Drug Discov. 2017;16(3):203-22.

11. Párrizas $M$, Novials A. Circulating microRNAs as biomarkers for metabolic disease. Best Pract Res Clin Endocrinol Metab. 2016;30(5):591-601.

12. Wang J, Liew OW, Richards AM, Chen Y-T. Overview of MicroRNAs in Cardiac Hypertrophy, Fibrosis, and Apoptosis. Int J Mol Sci 2016, 17(5).

13. Ortiz-Quintero B. Cell-free microRNAs in blood and other body fluids, as cancer biomarkers. Cell Prolif. 2016;49(3):281-303.

14. Arner P, Kulyté A. MicroRNA regulatory networks in human adipose tissue and obesity. Nat Rev Endocrinol. 2015;11(5):276-88.

15. Valadi H, Ekström K, Bossios A, Sjöstrand M, Lee JJ, Lötvall JO. Exosome-mediated transfer of mRNAs and microRNAs is a novel mechanism of genetic exchange between cells. Nat Cell Biol. 2007;9(6):654-9.

16. Mathivanan S, Ji H, Simpson RJ. Exosomes: extracellular organelles important in intercellular communication. J Proteomics. 2010;73(10):1907-20.

17. Barile L, Vassalli G. Exosomes: Therapy delivery tools and biomarkers of diseases. Pharmacol Ther. 2017;174:63-78.

18. Théry C, Zitvogel L, Amigorena S. Exosomes: composition, biogenesis and function. Nat Rev Immunol. 2002;2(8):569-79.

19. Ferguson SW, Nguyen J. Exosomes as therapeutics: The implications of molecular composition and exosomal heterogeneity. J Control Release. 2016;228:179-90.

20. Gallo A, Tandon M, Alevizos I, Illei GG. The majority of microRNAs detectable in serum and saliva is concentrated in exosomes. PLoS ONE. 2012;7(3):e30679.

21. Squadrito ML, Baer C, Burdet F, Maderna C, Gilfillan GD, Lyle R, Ibberson M, De Palma M. Endogenous RNAs modulate microRNA sorting to exosomes and transfer to acceptor cells. Cell Rep. 2014;8(5):1432-46.

22. Jiménez-Alesanco A, Marcuello $M$, Pastor-Jiménez $M$, López-Puerto $L$, Bonjoch $L$, Gironella $M$, Carrascal M, Abian J, de-Madaria E, Closa D. Acute pancreatitis promotes the generation of two different exosome populations. Sci Rep. 2019;9(1):19887.

23. Nakamaru K, Tomiyama T, Kobayashi S, Ikemune M, Tsukuda S, Ito T, Tanaka T, Yamaguchi T, Ando $\mathrm{Y}$, Ikeura T, et al. Extracellular vesicles microRNA analysis in type 1 autoimmune pancreatitis: Increased expression of microRNA-21. Pancreatology. 2020;20(3):318-24. 
24. Pinho AV, Chantrill L, Rooman I. Chronic pancreatitis: a path to pancreatic cancer. Cancer Lett. 2014;345(2):203-9.

25. Dweep H, Gretz N. miRWalk2.0: a comprehensive atlas of microRNA-target interactions. Nat Methods. 2015;12(8):697.

26. Bindea G, Mlecnik B, Hackl H, Charoentong P, Tosolini M, Kirilovsky A, Fridman W-H, Pagès F, Trajanoski Z, Galon J. ClueGO: a Cytoscape plug-in to decipher functionally grouped gene ontology and pathway annotation networks. Bioinformatics. 2009;25(8):1091-3.

27. Szklarczyk D, Gable AL, Lyon D, Junge A, Wyder S, Huerta-Cepas J, Simonovic M, Doncheva NT, Morris JH, Bork P, et al. STRING v11: protein-protein association networks with increased coverage, supporting functional discovery in genome-wide experimental datasets. Nucleic Acids Res. 2019;47(D1):D607-13.

28. Bader GD, Hogue CWV. An automated method for finding molecular complexes in large protein interaction networks. BMC Bioinformatics. 2003;4:2.

29. Chin C-H, Chen S-H, Wu H-H, Ho C-W, Ko M-T, Lin C-Y. cytoHubba: identifying hub objects and subnetworks from complex interactome. BMC Syst Biol. 2014;8(Suppl 4):11.

30. Storz P. Acinar cell plasticity and development of pancreatic ductal adenocarcinoma. Nat Rev Gastroenterol Hepatol. 2017;14(5):296-304.

31. Corcoran RB, Contino G, Deshpande V, Tzatsos A, Conrad C, Benes CH, Levy DE, Settleman J, Engelman JA, Bardeesy N. STAT3 plays a critical role in KRAS-induced pancreatic tumorigenesis. Cancer Res. 2011;71(14):5020-9.

32. Eser S, Reiff N, Messer M, Seidler B, Gottschalk K, Dobler M, Hieber M, Arbeiter A, Klein S, Kong B, et al. Selective requirement of PI3K/PDK1 signaling for Kras oncogene-driven pancreatic cell plasticity and cancer. Cancer Cell. 2013;23(3):406-20.

33. Saponara E, Visentin M, Baschieri F, Seleznik G, Martinelli P, Esposito I, Buschmann J, Chen R, Parrotta R, Borgeaud N, et al. Serotonin uptake is required for Rac1 activation in Kras-induced acinarto-ductal metaplasia in the pancreas. J Pathol. 2018;246(3):352-65.

34. Tao X, Chen Q, Li N, Xiang H, Pan Y, Qu Y, Shang D, Go VLW, Xue J, Sun Y, et al. Serotonin-RhoA/ROCK axis promotes acinar-to-ductal metaplasia in caerulein-induced chronic pancreatitis. Biomed Pharmacother. 2020;125:109999.

35. Kornmann M, Ishiwata T, Arber N, Beger HG, Korc M. Increased cyclin D1 expression in chronic pancreatitis. Pancreas. 1998;17(2):158-62.

36. Zhou L, Tan J-H, Cao R-C, Xu J, Chen X-M, Qi Z-C, Zhou S-Y, Li S-B, Mo Q-X, Li Z-W, et al. ATF6 regulates the development of chronic pancreatitis by inducing p53-mediated apoptosis. Cell Death Dis. 2019;10(9):662.

37. Gansauge F, Gansauge S, Eh M, Schlosser W, Ramadani M, Kern P, Beger HG. Distributional and functional alterations of immunocompetent peripheral blood lymphocytes in patients with chronic pancreatitis. Ann Surg. 2001;233(3):365-70. 
38. Gukovskaya AS, Pandol SJ. Cell death pathways in pancreatitis and pancreatic cancer. Pancreatology. 2004;4(6):567-86.

39. Puri S, Folias AE, Hebrok M. Plasticity and dedifferentiation within the pancreas: development, homeostasis, and disease. Cell Stem Cell. 2015;16(1):18-31.

40. Meda P, Bruzzone R, Knodel S, Orci L. Blockage of cell-to-cell communication within pancreatic acini is associated with increased basal release of amylase. J Cell Biol. 1986;103(2):475-83.

41. Ma C, Tian B, Wang J, Yang G-J, Pan C-S, Lu J-P. Metabolic characteristics of acute necrotizing pancreatitis and chronic pancreatitis. Mol Med Rep. 2012;6(1):57-62.

42. Petit $P$, Loubatières-Mariani MM. Potassium channels of the insulin-secreting $B$ cell. Fundam Clin Pharmacol. 1992;6(3):123-34.

43. Kirkegård J, Mortensen FV, Cronin-Fenton D. Chronic Pancreatitis and Pancreatic Cancer Risk: A Systematic Review and Meta-analysis. Am J Gastroenterol. 2017;112(9):1366-72.

44. Hong B, van den Heuvel APJ, Prabhu VV, Zhang S, El-Deiry WS. Targeting tumor suppressor p53 for cancer therapy: strategies, challenges and opportunities. Curr Drug Targets. 2014;15(1):80-9.

45. Gansauge S, Schmid RM, Muller J, Adler G, Mattfeldt T, Beger HG. Genetic alterations in chronic pancreatitis: evidence for early occurrence of $\mathrm{p} 53$ but not K-ras mutations. $\mathrm{Br} \mathrm{J}$ Surg. 1998;85(3):337-40.

46. Apte MV, Haber PS, Darby SJ, Rodgers SC, McCaughan GW, Korsten MA, Pirola RC, Wilson JS. Pancreatic stellate cells are activated by proinflammatory cytokines: implications for pancreatic fibrogenesis. Gut. 1999;44(4):534-41.

47. Jin G, Hong W, Guo Y, Bai Y, Chen B. Molecular Mechanism of Pancreatic Stellate Cells Activation in Chronic Pancreatitis and Pancreatic Cancer. J Cancer. 2020;11(6):1505-15.

48. Fitzner B, Müller S, Walther M, Fischer M, Engelmann R, Müller-Hilke B, Pützer BM, Kreutzer M, Nizze $\mathrm{H}$, Jaster R. Senescence determines the fate of activated rat pancreatic stellate cells. J Cell Mol Med. 2012;16(11):2620-30.

49. Pinho AV, Rooman I, Reichert M, De Medts N, Bouwens L, Rustgi AK, Real FX. Adult pancreatic acinar cells dedifferentiate to an embryonic progenitor phenotype with concomitant activation of a senescence programme that is present in chronic pancreatitis. Gut. 2011;60(7):958-66.

50. Sun J, Jiang Z, Li Y, Wang K, Chen X, Liu G. Downregulation of miR-21 inhibits the malignant phenotype of pancreatic cancer cells by targeting VHL. Onco Targets Ther. 2019;12:7215-26.

51. Wang L, Liang Y, Li P, Liang Q, Sun H, Xu D, Hu W. Oncogenic Activities Of UBE2S Mediated By VHL/HIF-1a/STAT3 Signal Via The Ubiquitin-Proteasome System In PDAC. Onco Targets Ther. 2019;12:9767-81.

52. Hu L-H, Ji J-T, Li Z-S. Potential application of miRNAs as diagnostic and therapeutic tools in chronic pancreatitis. J Cell Mol Med. 2015;19(9):2049-57.

53. Charrier A, Chen R, Chen L, Kemper S, Hattori T, Takigawa M, Brigstock DR. Connective tissue growth factor (CCN2) and microRNA-21 are components of a positive feedback loop in pancreatic stellate 
cells (PSC) during chronic pancreatitis and are exported in PSC-derived exosomes. J Cell Commun

Signal. 2014;8(2):147-56.

\section{Figures}

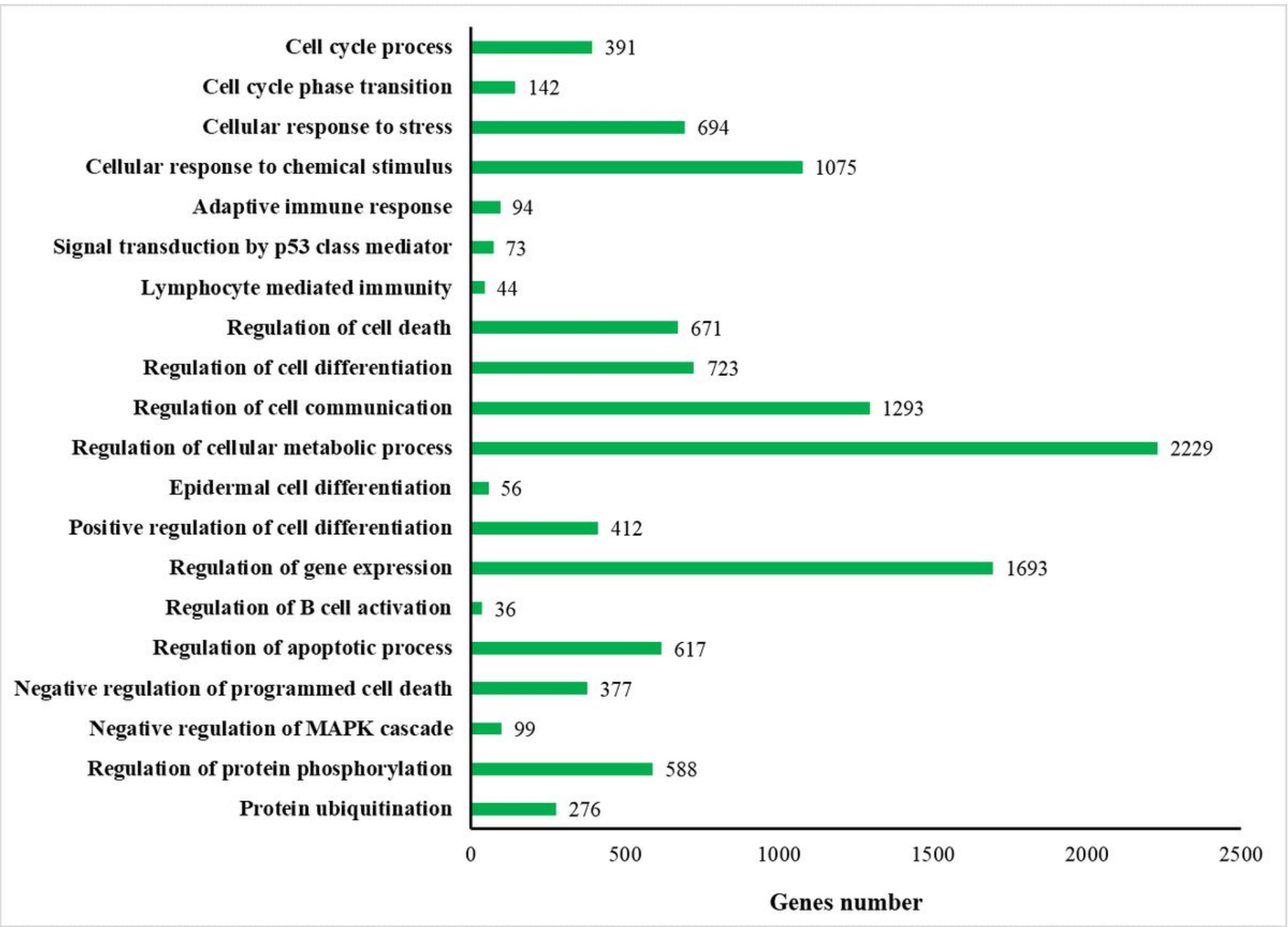

Figure 1

Go function annotation of miRNA target genes. Levels of GO terms increase from the upper to the lower. 


\section{Viral carcinogenesis}

\section{Taste transduction}

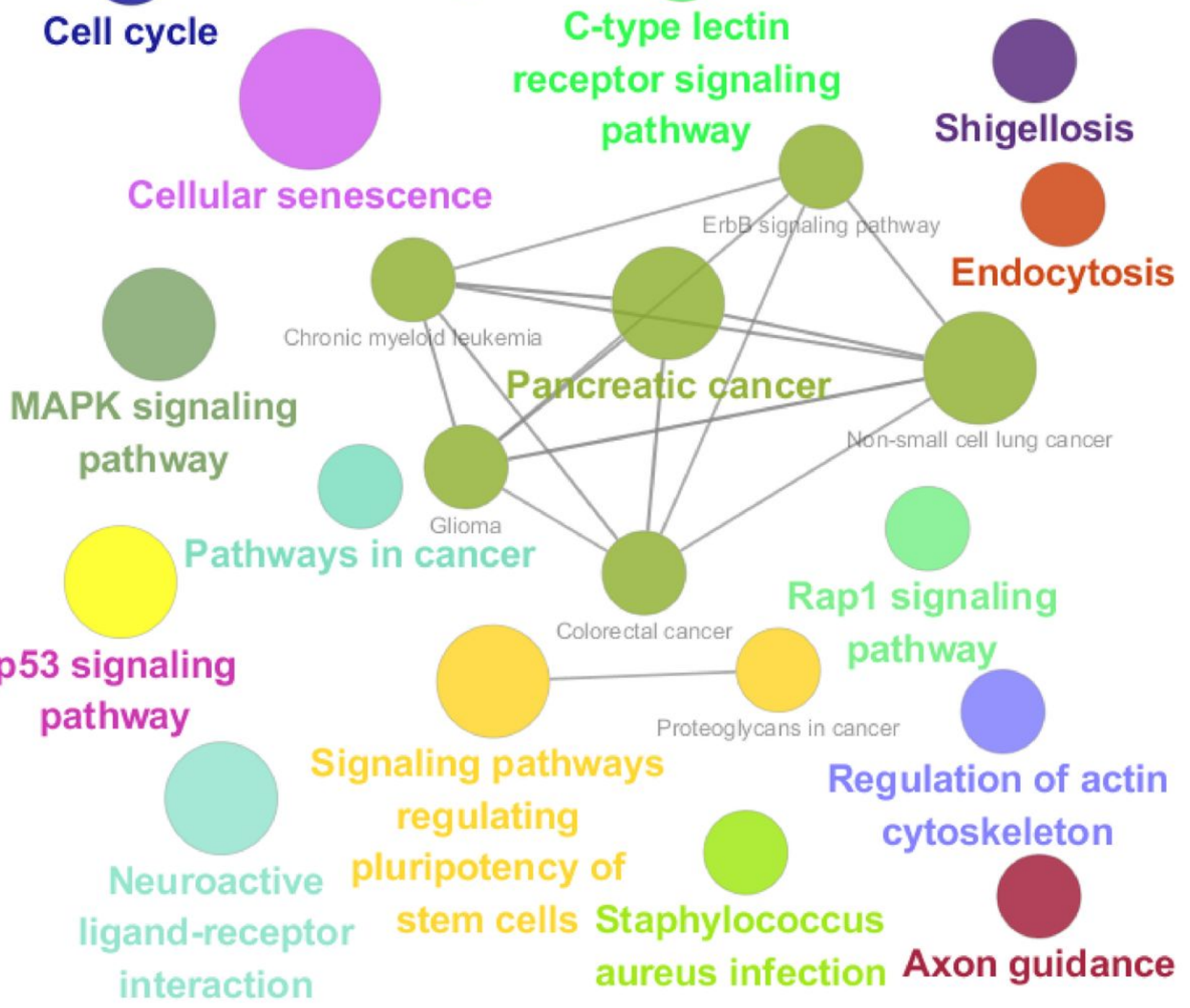

Figure 2

Functionally grouped network of KEGG pathways of serous DE-Exo-miRNA target genes. The node size stands for the pathway significance. The node size increases when the $p$-value of pathways decreases. 


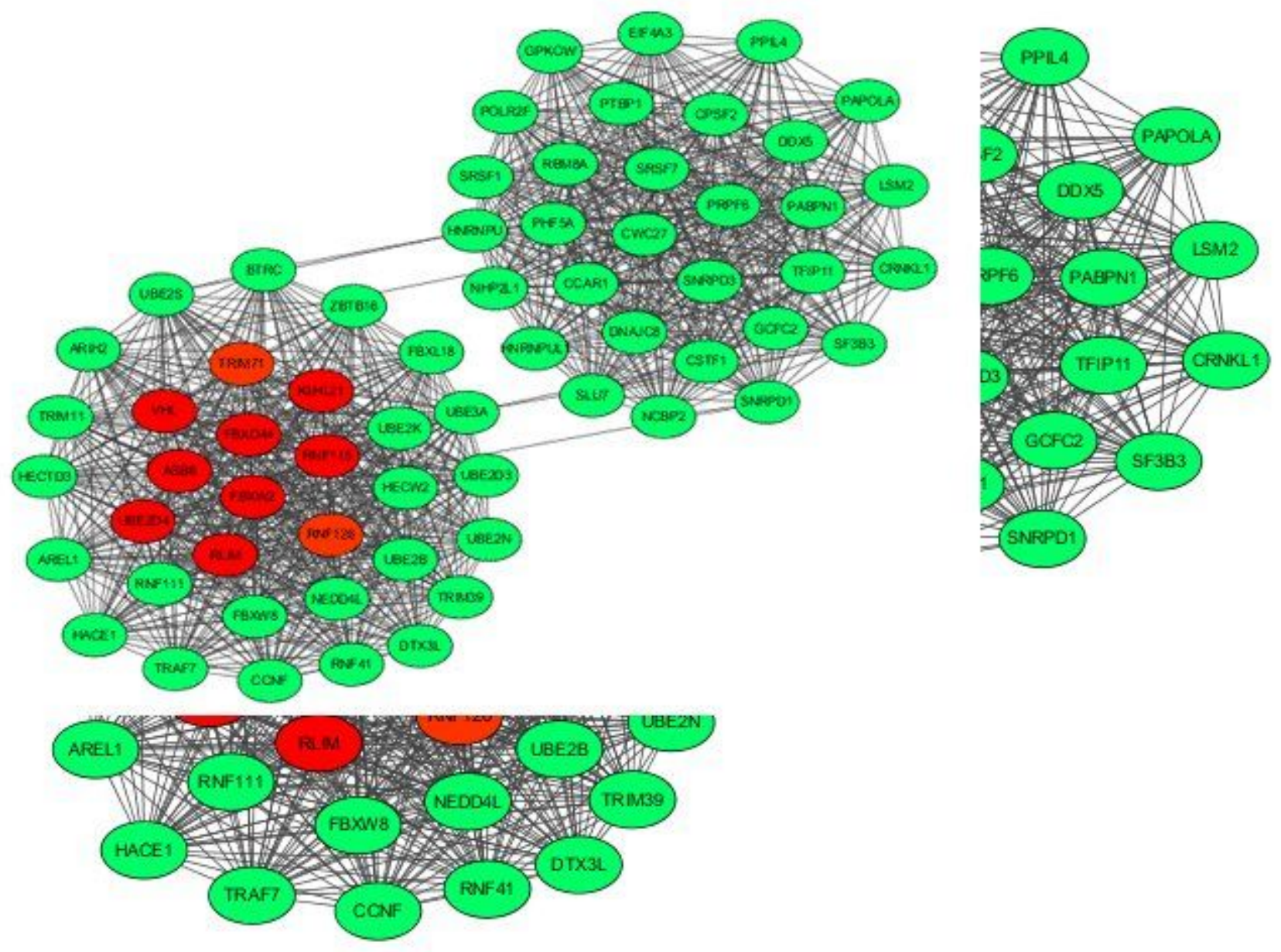

Figure 3

The submodule from the PPI network. The hub genes (nodes) are shown with red color. 


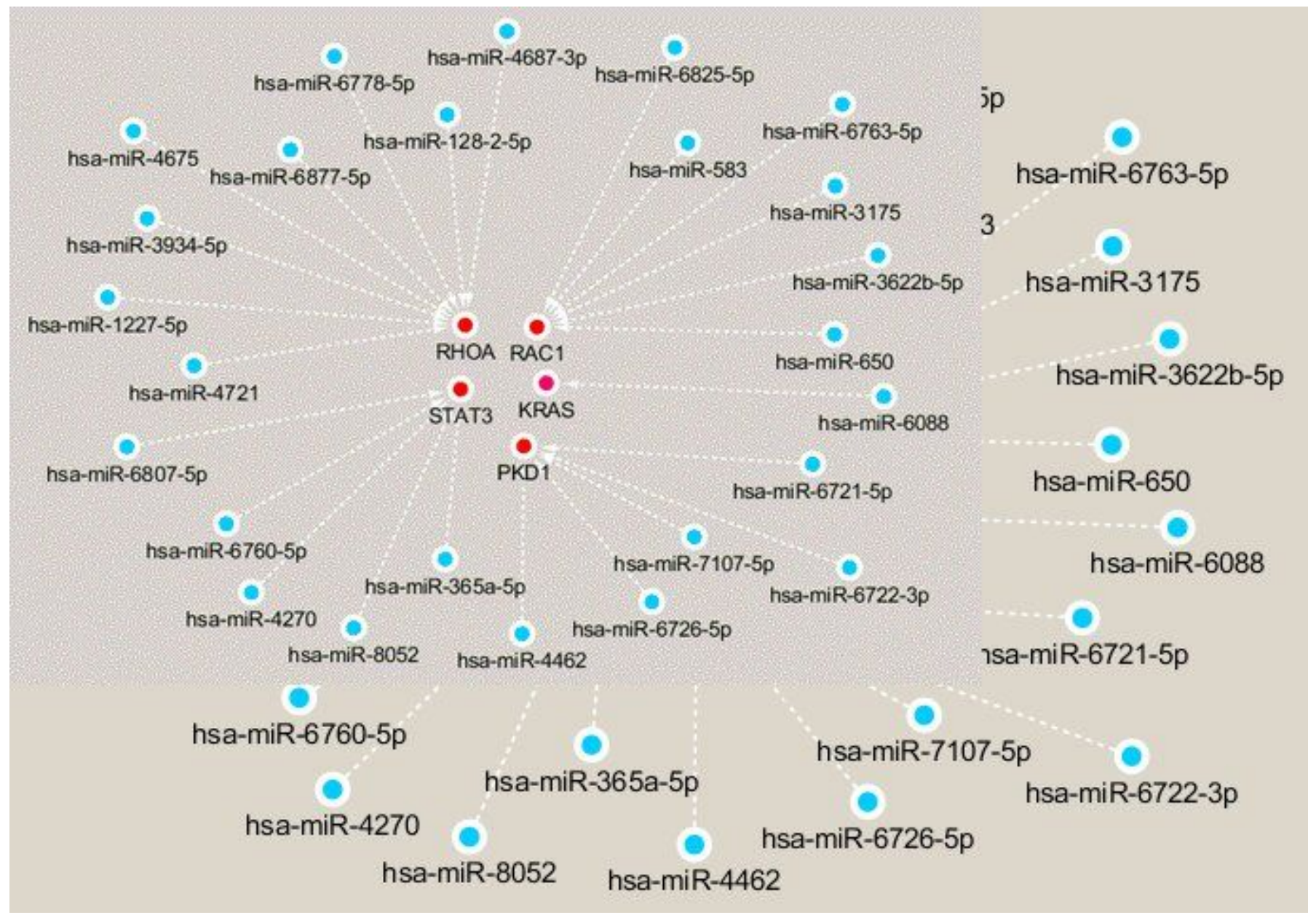

\section{Figure 4}

The Regulatory network of target genes associated with ADM and their miRNAs. The target genes (nodes) are shown with red color. The expression of these genes may be repressed by DE-S-Exo-miRNAs in the serum.

\section{Supplementary Files}

This is a list of supplementary files associated with this preprint. Click to download.

- Supplementarymaterial.xlsx 